\title{
COVID-19: Um Novo Desafio para a Cardiopatia na Gravidez
}

\section{COVID-19: A New Challenge in Pregnancy and Heart Disease}

\author{
Walkiria Samuel Avila1 ${ }^{\circledR 0}$ e Regina Coeli de Carvalho ${ }^{(1)}$ \\ Universidade de São Paulo Instituto do Coração, ${ }^{1}$ São Paulo, SP - Brasil \\ Hospital Geral de Fortaleza, Secretaria de Saúde do Estado do Ceará (SESA), ${ }^{2}$ Fortaleza, CE - Brasil
}

O novo coronavírus (SARS-CoV-2, do inglês Severe Acute Respiratory Syndrome Coronavirus-2) é o agente etiológico da COVID-19 (doença do novo coronavírus 2019), cujo surto foi declarado pandemia pela Organização Mundial da Saúde em 11 de março de 2020. ${ }^{1}$ Apesar de terem sido obtidos o isolamento, o sequenciamento genético e a análise estrutural do SARS-CoV-2, ainda não existem terapias especificas para COVID-19.

Os primeiros dados epidemiológicos indicam pior evolução e maior mortalidade para os pacientes com COVID-19 portadores de doenças crônicas, como cardiopatia e hipertensão arterial. O Ministério da Saúde do Brasil expandiu esse grupo de alto risco para grávidas, puérperas e mulheres após aborto. ${ }^{2}$

Evidências epidemiológicas anteriores sugerem fortemente que as mulheres grávidas têm um risco aumentado de doenças graves e morte por infecções virais durante pandemias, como a gripe. ${ }^{3}$

Alterações fisiológicas no período gestacional não aumentam apenas suscetibilidade a infecção viral, mas também a gravidade dessa doença (1). ${ }^{4-6}$ Durante a gravidez, a resposta imune predomina através das células T-helper 2 (Th2), que protegem o feto, mas tornam a mãe mais vulnerável a infecções virais, que são combatidas de forma mais eficaz pelas células Th1.

Grávidas infectadas com o vírus influenza, subtipo H1N1, e com dois outros coronavírus patogênicos, SARS-CoV (do inglês, severe acute respiratory syndrome coronavirus) e MERSCoV (do inglês, Middle East respiratory syndrome coronavirus), apresentaram alta morbimortalidade durante a gravidez e após o parto. Estimou-se que 90\% das grávidas com essas infecções virais evoluíram para insuficiência respiratória grave com complicações obstétricas, como aborto, parto prematuro e crescimento intrauterino restrito. Em grávidas infectadas com

\section{Palavras-chave}

Coronavírus; COVID-19; Pandemia; Gravidez; Gravidez de Alto Risco; Síndrome Respiratória Aguda Grave; Pneumonia; Hipertensão/prevenção e controle; Fatores de Risco; Morbidade; Mortalidade.

Correspondência: Walkiria Samuel Avila •

Rua Martiniano de Carvalho 864, cj 1107-1108

Bela vista - São Paulo CEP01321-000

E-mail: val walkiria@incor.usp.br

Artigo recebido em 22/05/2020, revisado em 27/05/2020, aceito em $27 / 05 / 2020$

DOI: https://doi.org/10.36660/abc.20200511
SARS-CoV ou MERS-CoV, foi relatada mortalidade materna de até $25 \%$, mas não houve relato de transmissão vertical transplacentária. ${ }^{8}$

Não há dados de que a gravidez aumente a suscetibilidade à COVID-19. Apesar de escassa evidência, parece que a COVID-19 durante a gravidez é menos grave do que as infecções pelo vírus influenza subtipo H1N1, SARS-CoV e MERS-CoV.

Estudos em grávidas infectadas com o SARS-CoV-2 são limitados a pequenas séries. Uma revisão sistemática ${ }^{9}$ de 108 grávidas com COVID-19 identificou tosse e febre como as principais queixas, presentes em quase $80 \%$ das mulheres, enquanto dispneia foi informada por apenas 12\%. Não há relato de morte materna. Das três pacientes graves que precisaram de ventilação mecânica, duas apresentavam obesidade (índice de massa corporal > 35) como fator de morbidade.

Outro estudo ${ }^{10}$ avaliando 116 grávidas com pneumonia por COVID-19 concluiu que as características clínicas da pneumonia das grávidas eram semelhantes àquelas da população geral. Atualmente não há evidência de que grávidas com COVID-19 estejam mais propensas a desenvolver pneumonia grave em comparação a não grávidas. Felizmente, não houve aumento de aborto espontâneo nem de parto prematuro natural, nem ainda evidência de transmissão vertical de SARS-CoV-2.

Transmissão perinatal de COVID-19: Devemos nos preocupar? Dos 75 recém-nascidos de mães com COVID-19, apenas um foi positivo para o vírus e apresentou evolução clínica satisfatória com leve alteração nas enzimas hepáticas. ${ }^{9}$ Entretanto, alguns bebês que testaram negativo para COVID-19 apresentaram linfocitopenia e achados radiológicos de pneumonia, e um apresentou coagulação intravascular disseminada. Todos os bebês se recuperaram totalmente. ${ }^{11,12}$ Em vista disso, não se pode excluir a possibilidade de uma resposta subclínica de fetos e recém-nascidos à infecção materna, nem transmissão vertical transplacentária. Portanto, recomenda-se o monitoramento cuidadoso de recémnascidos de mães com COVID-19.

As grávidas com cardiopatia ou hipertensão arterial e infecção pelo SARS-CoV-2 devem ser consideradas de maior risco para mortalidade? O documento da Sociedade Brasileira de Cardiologia para conduta em doenças cardíacas na gravidez, ${ }^{13}$ que inclui protocolos de cuidados, estratégias de tratamento e prevenção de complicações cardíacas durante a gravidez, contribuiu para a redução da mortalidade materna no Brasil. No entanto, enfrentamos a emergência da COVID-19, uma doença que compromete essas conquistas. A continuidade das pesquisas clinicas e novas abordagens 


\section{Editorial}

\section{Tabela 1 - Impacto das alteraçôes fisiológicas cardiovasculares e respiratórias em grávidas cardiopatas com SARS-CoV-2}

\begin{tabular}{l}
\hline • Desequilibrio do sistema imune materno: atenuação Th1 e dominância Th2 - Risco de infecções virais \\
\hline - Aumento do consumo de oxigênio: hipoventilação, apneia ou comprometimento da troca gasosa - Hipoxemia materna e fetal \\
\hline • Diminuição da capacidade residual funcional pulmonar materna $(10 \%-25 \%)$ - Hipoxemia \\
\hline • Hiperemia e edema de vias aéreas superiores - Dificuldade na intubação endotraqueal \\
\hline • Aumento do Volume das mamas e necessidade de intubação e sequência rápida devido a esvaziamento gástrico retardado - Risco de aspiração \\
\hline • Redução da resistência vascular sistêmica - Hipotensão materna e hipoxemia \\
\hline • Aumento da frequência cardíaca e do volume sistólico - Insuficiência cardíaca \\
\hline -Ventilação Mecânica \\
\hline Hiperventilação e alcallose respiratória - Vasoconstrição uterina \\
\hline Hipoventilação e hipercapnia - Acidose respiratória fetal \\
\hline PaO2 materna deve ser mantida $\geq 70$ mmHg - Adequada oxigenação fetal \\
\hline Risco aumentado de tromboemlismo \\
\hline Aumento nos fatores de coagulação (V, VIII, X e von Willebrand) \\
\hline Redução nos níveis de proteína S \\
\hline Compressão uterina da veia cava inferior e da veia ilíaca esquerda \\
\hline Trauma local da veia cava inferior e da veia ilíaca esquerda durante o parto \\
\hline Após parto cesárea
\end{tabular}

Tabela 2 - COVID-19 / Cardiopátia / Gravidez - Características e diagnóstico diferencial da triade

\begin{tabular}{|c|c|c|c|}
\hline & Covid-19 & Cardiopatia & Gravidez normal \\
\hline Sintomas & $\begin{array}{l}\left.\text { Febre (>37,8 }{ }^{\circ} \mathrm{C}\right) \text {, mialgia, fadiga, } \\
\text { anorexia, dor de garganta, congestão } \\
\text { nasal e conjuntival, tosse, dispneia, } \\
\text { anosmia, anorexia, odinofagia, náusea, } \\
\text { vômito, diarreia, dor abdominal. }\end{array}$ & $\begin{array}{l}\text { Dispneia, palpitação, dor torácica, } \\
\text { sincope, hemoptise, fadiga, edema } \\
\text { de membros inferiores, ortopneia, } \\
\text { tosse seca }\end{array}$ & $\begin{array}{l}\text { Náusea, vômito, edema, dispneia, } \\
\text { fadiga, palpitações, tontura, epistaxe, } \\
\text { rinite gestacional, cefaleia, dor } \\
\text { abdominal }\end{array}$ \\
\hline $\begin{array}{l}\text { Aparecimento dos sintomas e } \\
\text { idade gestacional }\end{array}$ & $\begin{array}{l}\text { Qualquer idade gestacional ou } \\
\text { puerpério }\end{array}$ & $\begin{array}{l}\text { Em geral no } 2 \text { e } 3 \text { trimestre e } \\
\text { puerpério }\end{array}$ & Qualquer idade gestacional \\
\hline História & Sem cardiopatia prévia & Cardiopatia prévia & Sem cardiopatia prévia \\
\hline Exames laboratoriais & $\begin{array}{l}\text { RT-PCR swab nasofaringeo positivo } \\
\text { para COVID-19 } \\
\text { Linfocitopenia } \\
\text { Aminotransferases (ALT/AST): } \\
\text { elevadas } \\
\text { ureia/creatinina alteradas } \\
\text { Dimero d: elevado }\end{array}$ & $\begin{array}{l}\text { Peptídeo natriurético tipo B: niveis } \\
\text { altos }\end{array}$ & $\begin{array}{l}\text { Dimero d: normal ou levemente } \\
\text { elevado }\end{array}$ \\
\hline Exames de imagem & $\begin{array}{l}\text { Ecocardiograma: normal } \\
\text { Raio-X de tórax: com ou sem } \\
\text { alteração } \\
\text { Tomografia de tórax: opacidade em } \\
\text { "vidro fosco" }\end{array}$ & $\begin{array}{l}\text { Ecocardiograma: lesão } \\
\text { cardíaca estrutural } \\
\text { Alteração de raio- X/tomografia de } \\
\text { torax: cardiomegalia e/ou congestão } \\
\text { pulmonar }\end{array}$ & $\begin{array}{l}\text { Ecocardiograma: normal } \\
\text { Raio-X de tórax: normal }\end{array}$ \\
\hline
\end{tabular}

RT-PCR: em inglês: reverse transcription polymerase chain reaction assay; ALT: alanina aminotransferase; AST: aspartato aminotransferase.

para integrada para o subgrupo de grávidas com cardiopatia ou hipertensão afetadas pelo SARS-CoV-2 são mandatórias.

A perspectiva de desfecho otimista para a combinação de gravidez e infecção pelo SARS-CoV-2 torna-se incerta em mulheres com cardiopatia ou distúrbios hipertensivos, pois essas duas condições cardíacas isoladamente representam as principais causas de mortalidade materna e fetal na gravidez.
É importante que a suspeita clínica de COVID-19 em mulheres grávidas com doença cardíaca seja descartada. Doenças cardíacas e COVID-19 têm sintomas em comum, o que pode levar a erro diagnóstico (Tabela 2). Em vista disso e considerando a atual pandemia, testes para SARS-CoV-2 devem fazer parte das boas práticas de triagem para grávidas com doença cardíaca.

As Alterações fisiológicas no sistema cardiorrespiratório devidas à gravidez não aumentam a suscetibilidade à 
infecção pelo vírus, mas podem piorar o desfecho materno ${ }^{4-6}$. As alterações respiratórias na gravidez levam a redução da capacidade pulmonar total e da complacência torácica no final da gestação. Além disso a hipóxia materna decorrente da hipoventilação e do comprometimento nas trocas gasosas reduz a oferta de oxigênio para o feto, e consequentemente, morte intra-uterina. Nesse contexto, a pneumonia da COVID-19 progride rapidamente de consolidação pulmonar focal para difusa bilateral, predispondo a grave insuficiência respiratória hipoxêmica.

COVID-19 pode levar a injúria cardíaca por múltiplos mecanismos, resultando em resposta inflamatória extrema com lesão endotelial e miocardite. ${ }^{14} \mathrm{Na}$ gravidez e no período pós-parto, insuficiência cardíaca aguda deve ser considerada em algumas circunstâncias, como cardiomiopatia periparto, miocardite viral e edema pulmonar não cardiogênico. Edema pulmonar é também visto em grávidas saudáveis em decorrência de importantes alterações no volume intravascular durante o trabalho de parto e após o parto. Da mesma forma, alterações hemodinâmicas na gestação causam aumento no gradiente da válvula mitral estenótica e pode levar a congestão pulmonar. A cardiopatia congênita cianótica, as lesões obstrutivas do lado esquerdo do coração ou grave disfunção ventricular sistólica apresentam maior risco de complicações cardíacas em mulheres grávidas. A queda da resistência vascular sistêmica piora a hipoxemia em mulheres grávidas com hipertensão pulmonar e com tetralogia de Fallot não corrigida.

Coagulopatia sistêmica é um aspecto crítico de morbimortalidade na COVID-19. ${ }^{14}$ O estado de hipercoagulabilidade da gravidez eleva o risco de tromboembolismo em mulheres cardiopatas. Nesse cenário, a combinação de COVID-19 e prótese valvular mecânica ou fibrilação atrial na doença valvar reumática aumenta o risco de eventos tromboembólicos em mulheres grávidas. Vale ressaltar que, a cada trimestre da gestação, os níveis de dímero-D aumentam, fato que deve ser considerado na interpretação para diagnóstico de tromboembolismo pulmonar.

Inflamação sistêmica e coagulopatia na COVID-19 elevam o risco de ruptura da placa aterosclerótica e infarto agudo do miocárdio. ${ }^{14}$ A significativa implicação de infecção pelo SARSCoV-2 para o sistema cardiovascular é evidenciada por injúria miocárdica aguda (altos níveis de troponina I ultrassensível e/ ou novas anormalidades no ECG/ecocardiograma), arritmias cardíacas complexas e parada cardíaca. Durante a gravidez, síndrome coronariana aguda não é comum. Entretanto, infecções, em especial no pós-parto, são fatores de risco para infarto do miocárdio. As causas mais frequentes de infarto do miocárdio na gravidez são dissecção espontânea de artéria coronária, aterosclerose, trombose coronariana e artérias normais na angiografia com comprometimento da microcirculação coronariana.

Estudos recentes mostraram que a enzima de conversão da angiotensina 2 (ECA2) é um receptor funcional de SARS-CoV-2..$^{15} \mathrm{O}$ sistema renina-angiotensina é o principal responsável pela regulação da pressão arterial e a ECA2 tem papel crítico no controle da fisiologia cardiovascular em grávidas. Angiotensina-(1-7) é significativamente elevada em grávidas saudáveis quando comparadas a não grávidas. Na pré-eclâmpsia, os níveis plasmáticos de angiotensina (1-7) são reduzidos e a angiotensina II plasmática é consistentemente elevada, o que contribui para o desenvolvimento de hipertensão nessas gestantes. Além disso, grávidas com hipertensão crônica apresentam maior risco de pré-eclâmpsia ou síndrome HELLP. Contudo, a relação entre regulação positiva de ECA2 e SARS-CoV-2 na gravidez requer estudos adicionais.

Por fim, atualmente, não há dados sobre o desfecho da gravidez em pacientes com cardiopatia ou hipertensão arterial e COVID-19. Entretanto, essas pacientes têm que ser consideradas um grupo de alto risco.

Devido à falta de terapêutica específica e de vacina para COVID-19, precisamos estar preparados para prevenir e tratar complicações cardiovasculares na gestação. ${ }^{13}$ Cuidado integrado e multidisciplinar deve visar à otimização da terapia, à orientação das pacientes quanto aos riscos da COVID-19 e ao seu tratamento em uma eventual infecção por SARS-CoV-2.

As graves consequências da COVID-19 somadas às complicações de grávidas com cardiopatia ou hipertensão arterial podem determinar pior desfecho materno e prognóstico incerto.

\section{Referências}

1. World Health Organization. (WHO) Coronavirus disease (COVID-19) Pandemic. [Cited in 2020 Apr 23] Available from: https://www.who.int/ emergencies/diseases/novel-coronavirus-2019.

2. Brasil.Ministério da Saúde. Secretaria de Atenção Especializada à Saúde. Protocolo de manejo clínico da Covid-19 na Atenção Especializada. [Citado em 19 abr 2020] Disponível em: https://portalarquivos.saude. gov.br/images/pdf/2020/April/14/Protocolo-de-Manejo-Cl--nico-parao-Covid-19.pdf

3. Meljer WJ, van Noortwijk AG, Bruinse HW, Wensing AM. Influenza virus infection in pregnancy. A review. Acta Obstet Gynecol Scand. 2015 Aug;94(8):797-819.

4. Sanghavi M, Rutherford JD. Cardiovascular physiology of pregnancy. Circulation. 2014;130(12):1003-8.

5. Cui C, Yang S, Zhang J, Wang G, Huang S, Li A, et al. Trimester-specific coagulation and anticoagulation reference intervals for healthy pregnancy. Thromb Res. 2017 Aug;156:82-6.

6. Hegewald MJ. Respiratory physiology in pregnancy. Clin Chest Med $2011 ; 32(1): 1-13$.

7. Kwon JY, Romero R, Mor G. New insights into the relationship between vira infection and pregnancy complications. Am J Reprod Immunol. 2014 May; 71(5):387-90.

8. Schwartz DA, Graham AL. Potential Maternal and Infant Outcomes from (Wuhan) Coronavirus 2019-nCoV Infecting Pregnant Women: Lessons from SARS, MERS, and Other Human Coronavirus Infections. Viruses. 2020 Feb 10;12(2):194. 


\section{Editorial}

9. Zaigham M, Andersson O. Maternal and perinatal outcomes with COVID-19 : A systematic review of 108 pregnancies Acta Obstetric Gynecol Scand. 2020;00:1-7

10. Yan J, Guo J, Fan C, Juan J, Yu X, Li J, et al. Coronavirus disease 2019 COVID-19) in pregnant women: a report based on 116 cases Am J Obstetr Gynecol. 2020

11. Zhu H, Wang L, Fang C, Peng S, Zhang L, Chang G, et al. Clinical analysis of 10 neonates born to mothers with 2019-nCoV pneumonia. Transl Pediatr. 2020;9(1):51-60.

12. Fan C, Lei D, Fang C, Li C, Wang M, Liu Y, et al. Perinatal transmission of COVID_19 associated SARS-Cov2: Should we worry? Clin Infect Dis,2020 Mar 1y.ciaa226. Online ahead of print
13. Avila WS, Alexandre ERG, Castro ML, Lucena AJG, Marques-Santo C, Freire CMV, et al. Brazilian Cardiology Society Statement for Management of Pregnancy and family Planning in women with Heart Disease-2020- Arq Bras Cardiol. 2020;114(5):849-942

14. Long B, Brady WJ, Koyfman A, Gottlieb M. Cardiovascular complications in COVID-19, Am J Emerg Med. 2020 Apr 18;S0735-6757(20)30277-1.

15 Chen L, Li X, Chen M, Feng Y, Xiong C, The ACE2 expression in human heart indicates new potential mechanism of heart injury among patients infected with SARS-CoV-2 Cardiovasc Res. 2020 May 1;116(6):1097-1100. 